\title{
Laser Direct Write Fabrication of Meta-Antennas for Electro-Optic Conversion
}

\author{
A. T. Smith ${ }^{1}$, D. Simonson ${ }^{2}$, N. A. Charipar ${ }^{2}$, A. Piqué ${ }^{2}$ \\ ${ }^{1}$ Nova Research, Inc. \\ 1900 Elkin Street, Suite 230, Alexandria, Virginia 22308 \\ E-mail: andrew.t.smith.ctr@nrl.navy.mil \\ ${ }^{2}$ Naval Research Laboratory, Materials Science and Technology Division, Code 6364, \\ 4555 Overlook Ave. SW, Washington, DC 20375, USA
}

\begin{abstract}
For electro-optic modulators, traveling wave designs are implemented due to the long interaction area necessary to efficiently modulate a signal with low voltages as a result of $\mathrm{LiNbO}_{3}$ 's modest electro-optic coefficient $\left(\mathrm{r}_{33}=30 \mathrm{pm} / \mathrm{V}\right)$. However, with metamaterial inspired, electrically small resonator antennas, high field enhancements can be produced which allow the modulation of optical signals directly from the antennas, bypassing the need for a separate modulator [1]. For this work, Laser Direct Write (LDW) proved to be a useful tool in fabricating various meta-antenna array structures. The additive techniques of laser direct write, coupled with more traditional micromachining, have allowed the quick fabrication of these resonator meta-antennas so that the desired specifications, including resonant frequency and field enhancement, can be tested quickly and appropriate updates to the design can be made. This allows a designer to shift the resonant frequency and increase the field enhancement necessary to get the required device performance. The Laser Induced Forward Transfer (LIFT) technique was used initially for lower frequency designs (X-band); however, Laser Decal Transfer (LDT) was used subsequently for designs with higher frequencies by employing a digital mirror device to modulate the image of the beam. The resulting designs showed good fabrication consistency in terms of resonant frequency and comparable field enhancement to resonators fabricated through traditional lithography.
\end{abstract}

DOI:10.2961/jlmn.2012.03.0013

Keywords: Laser Direct Write, Electro-optic Conversion, Metamaterials, Electrically Small Antennas

\section{Introduction}

Laser Direct Write or LDW encompasses a group of laser-based processes for rapidly prototyping structures by adding, removing, or modifying materials. The flexibility provided by these processes allow for the quick realization of designed structures that would normally take up to weeks using standard lithography which requires considerable time for mask fabrication. For creating proofs of concept or iterating designs and prototypes, this decreased design to production time is invaluable for both industry and research purposes.

One of the additive Laser Direct Write processes is Laser Induced Forward Transfer (LIFT), which is a process that uses a pulsed laser for transferring materials from a donor substrate to a target substrate with micron level precision. The process allows for great flexibility in that a wide variety of materials can be effectively printed over any surface including battery electrode materials, conductors for sensors or electronic components, and various other materials of interest [2-4].

In many cases, the material transferred is made up of nano or micro scale particles that are dispersed in a particular solvent. After transfer the substrate is usually placed through a curing process in order to remove any remaining solvent as well as to sinter the particles together. The benefits of moving to smaller particle size generally are better feature definition as well as a lower curing temperature needed to get effective sintering.

Another LDW process that can be used for microfabrication is Laser Decal Transfer (LDT). This process is similar in principle to LIFT however it takes advantage of the high viscosity of a nanopaste in that the transferred material keeps the shape of the incident beam [5]. By using an aperture or other beam shaping device, it is possible to fabricate complex shapes with a single laser shot. This allows for increased production speed as the design does not need to be built up pixel by pixel, rather the unit structure is fabricated in a more parallel manner. The basic setup for this system is shown in Figure 1. Generally, this process is carried by using apertures to image the beam, but recently a spatial light modulator was implemented for shaping the beam instead [6]. This permits on the fly changes to the desired beam shape and thus the transferred structure shape. The added flexibility granted by this device enables the creation of much more complex structures, more rapid design iteration, and easier implementation.

In this paper we will describe the use of these two Laser Direct Write techniques for the creation of electrically small antennas that can directly modulate optical signals. 


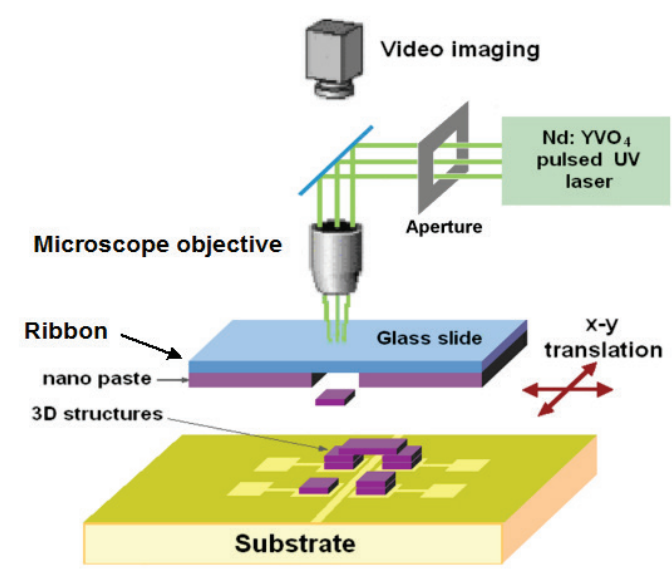

Figure 1 The basic setup for the Laser Decal Transfer process where an imaged laser beam transfers viscous nanopaste that maintains the shape of the incident beam.

\section{Background}

Optical networks and systems are growing rapidly in scope and prevalence due to their many advantages over traditional electronic systems. Some of these benefits include increase bandwidth and lower losses as well as decreased size, weight and power requirements. Since electronic based systems are a well established technology that are not likely to be phased out any time soon, it is necessary to have interfaces that can convert electric signals to optical signals and vice-versa. Currently, photodiodes provide a relatively mature means of converting optical signals to electronic signals; however, conversion in the opposite direction is much less developed. Considerable research effort is being directed into the creation of high speed, low loss modulators in order to keep up with the demand for increased bandwidth.

There are a few mechanisms available for opto-electric conversion, one of the more prevalent being the Pockel's effect, where the application of an electric field to an electro-optic material proportionally changes the refractive index of that material. The change of a material's refractive index in proportion to the applied field strength is referred to as its electro-optic coefficient $\left(\mathrm{r}_{33}\right)$. The predominant material for electro-optic conversion is lithium niobate $\left(\mathrm{LiNbO}_{3}\right)$ which has a coefficient $\mathrm{r}_{33}=30 \mathrm{pm} / \mathrm{V}$. For a given coefficient value, the strength of the modulated optical signal is heavily dependent on the applied field strength and the distance the electric field interacts with the optical signal.

For many platforms, space is a very serious design constraint, thus integrating and miniaturizing systems is a high priority. For antennas and other RF applications, electrically small antennas provide an avenue to achieve this necessary miniaturization. Yet, for integration with an optical network, a separate modulator is still necessary and detracts from the space savings of a small antenna. A proposed solution to this involves integrating the modulator and antenna into a single unit by using a high $\mathrm{Q}$ resonator antenna that has high enough field enhancement to effi- ciently modulate the optical signal [1] as illustrated in Figure 2 .

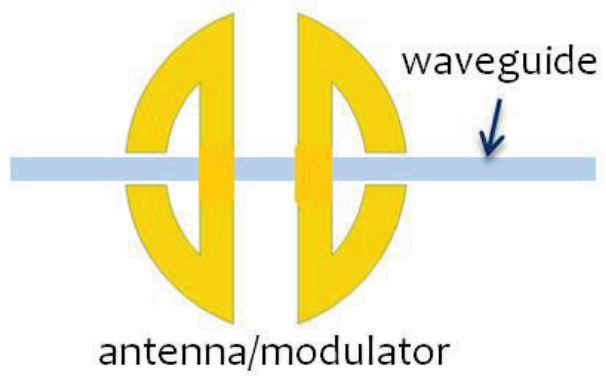

Figure 2 An illustration of an antenna directly modulating an optical signal by having a gap in the antenna where e-fields will modulate the optical signal in the waveguide within the gap.

The particular designs that are proposed for the integrated antenna and modulator, are inspired from negative permittivity spherical antennas [7] and are illustrated in Figure 3. Both designs have a gap where field strength will be enhanced due to the resonance of the structure. Placing the waveguide beneath this gap will allow the incident RF to modulate the optical signal in the waveguide via the resonator antenna. By allowing for high field enhancement, the field strength will be high enough to reach acceptable modulation levels.

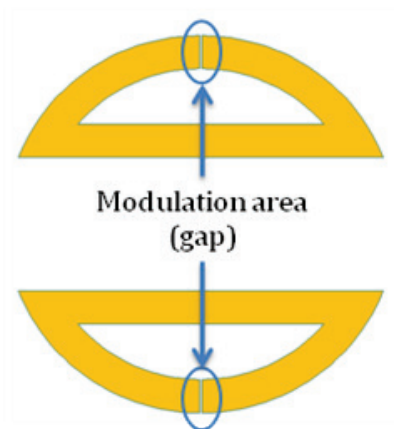

(a)

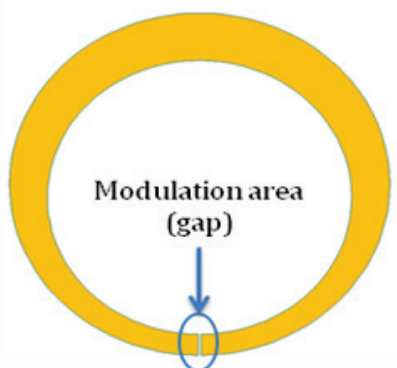

(b)
Figure 3 The two designs investigated in this work as integrated antennas and electro-optic modulators are the (a) D-Ring and (b) Crescent Ring.

\section{Experimental}

The system used for the LIFT process, as well as the LDT fabrication performed with apertures, was a frequency tripled Nd: $\mathrm{YVO}_{4}$ that has an operating wavelength of 355 $\mathrm{nm}$ and fired at $10 \mathrm{kHz}$. The optical path imaged an aperture through a 10x objective with NA 0.25 . The energy and timing of the pulses were controlled using an acousto-optic modulator. A CCD camera is place along the focusing axis in order to image the receiving substrate or the ribbon/receiving substrate interface. For the LDT fabricated transfers using the Digital Mirror Device, a Nd:YAG 532 $\mathrm{nm}$ Quantel laser was used with a maximum rep rate of 50 Hz. The Digital Mirror Device was a Texas Instruments Digital Light Projector Discovery 4100 .7" XGA. A FX-50 Ophir Beam Profiler was used to evaluate the beam power distribution after it has been imaged by an aperture or by the DMD. 
The nanoink used was Harima Nanopaste NPS consisting of silver nanoparticles diluted using AF-5 solvent at a 7\% wt concentration. The ink was applied to a quartz wafer using a wire coater and then spun coat at $2000 \mathrm{rpm}$ for 30 seconds to help disperse the nanoink uniformly. The ribbon preparation for the LDT consisted of doctor blading undiluted Harima Nanopaste into wells $1.5-2 \mu \mathrm{m}$ deep in glass. The ribbon was allowed to air dry for 30 minutes to an hour before transferring.

For the LIFT fabricated antennas, the receiving substrate was a 3" X-Cut lithium niobate wafer with titanium diffused waveguides $8 \mu \mathrm{m}$ in width. The antennas made using LDT were transferred onto a $1 \mathrm{~cm} \mathrm{x} 1 \mathrm{~cm} \mathrm{Si} \mathrm{(p-type)}$ substrate. The alignment was made using fiducials that were diffused into the lithium niobate in the same step that the waveguides were fabricated.

Optical microscopy was used to inspect the antennas before and after curing. A contact profilometer (KLA Tencor P-10) was used to measure the height and thickness of the cured samples. An SEM was also used to get high magnification images of the LDT transfers for the higher frequency antennas.

\section{Discussion}

For fabricating theses structures using Laser Direct Write techniques, several key design aspects had to be considered which provided particular challenges.

1) The antenna interaction area gap had to be $10 \mu \mathrm{m}$ wide and maintain that width throughout its length.

2) The antenna had to be placed so that the interaction area gap was perfectly aligned with the waveguide

3) The surface and waveguide of the lithium niobate should not be damaged.

4) The laser transferred metal layer had to be approximately $2 \mu \mathrm{m}$ or greater in thickness.

Since the printed material is a relatively low viscosity ink in LIFT, it tends to flow on the substrate after it is transferred. Slight variations in ribbon and ink preparation can change the wetting behavior and thus how the edges of a structure are defined. It was determined that a "trimming" step was needed to remove the excess ink that moved outside of the desired dimensions, especially the gap for the waveguide, in order to maintain the required tolerances. It was found that the uncured ink could be removed using the same fluence levels used for the actual transfer of the ink to the substrate

Though it was determined necessary to use ablative means to achieve the desired dimensions, the surface of the lithium niobate wafer, and the waveguides in particular, needed to be protected from any damage or surface modification from the laser. In order to guarantee the protection of the lithium niobate, two resist layers were applied on top of the lithium niobate wafer. The first layer was a Lift-Off Resist (LOR), which can be processed at the higher temperatures necessary to cure the ink. The second layer was a positive photoresist (Shipley 1818) that allowed for exposing the desired shape of the antenna structure. Both resists could then be developed using Microposit MF-319 leaving wells in the same shape as the antenna. Once the LOR layer was defined, the photoresist layer was removed by an acetone immersion. The thickness of the LOR layer was determined based on how thick the metal layer needed to be.

Once preparation of the resist layer was complete, the silver nanoink was transferred using the LIFT process. and then trimmed to the desired dimensions. Finally, the sample was placed in an oven and ramped up to $180^{\circ} \mathrm{C}$ at a rate of 3 degrees/minute to avoid thermal stress on the lithium niobate. Then the LOR layer was removed and the sample was placed back into the oven and ramped up to $230{ }^{\circ} \mathrm{C}$ to achieve maximum curing and conductivity. Figure 4 shows a flowchart for this process, while the fully cured antennas can be seen in Figure 5.

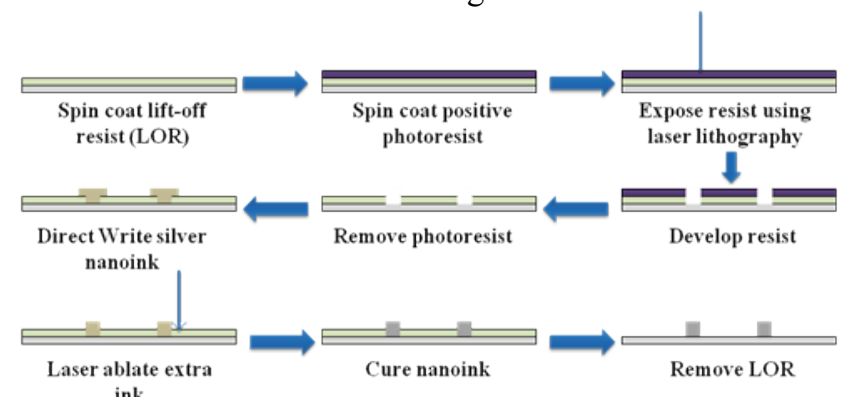

Figure $4 \mathrm{~A}$ flow chart of the process used to fabricate the antennas using the LIFT process

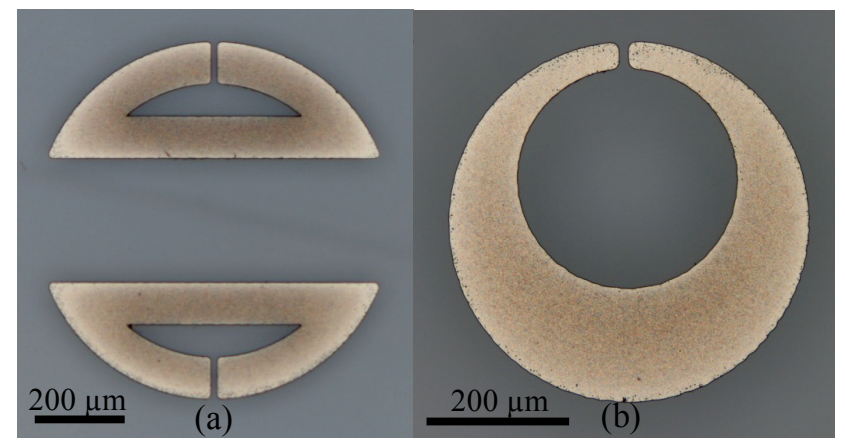

Figure 5 Optical microscopy images of the cured $30 \mathrm{GHz}$ versions of the (a) D-Ring and (b) Crescent Ring antennas

A second design for the $10 \mathrm{GHz}$ D-Ring was created in order to increase the modulation efficiency of the antenna. As mentioned earlier, the modulation of the optical signal correlates with both the applied field to the electro-optic material, as well as the length of the interaction of the optical signal with the E-field. The second iteration of the design incorporated a longer interaction area so that the optical signal would be more effectively modulated. An illustration as well as a picture of the cured second iteration antenna can be seen in Figure 6. The lengthening of the interaction area had the effect of decreasing the size of the antenna. Since the antennas are electrically small, the lumped circuit element model works well in describing the antennas behavior. Thus the lengthened feature had the effect of increasing the overall capacitance of the antenna, which would decrease the antenna's size for a desired resonant frequency. 


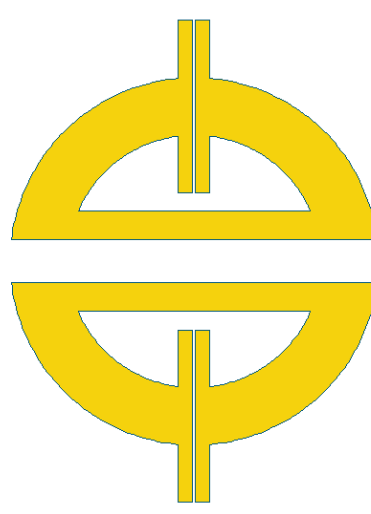

(a)

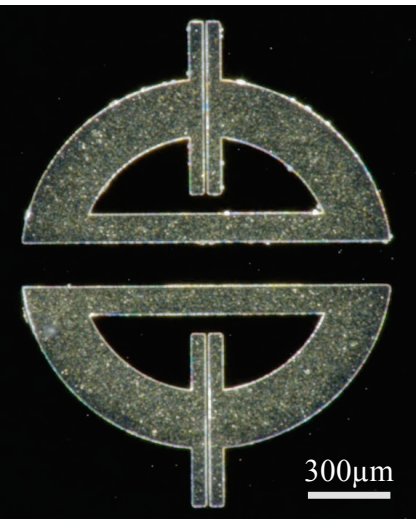

(b)
Figure 6 An (a) illustration and (b) an image of a cured D-Ring with a modified design.

For higher frequency designs, the antenna sizes were reduced accordingly making them less than $250 \mu \mathrm{m}$ in diameter. This falls within the appropriate dimensions to be fabricated using LDT.

For initial LDT fabrication, an aperture was created to image the beam and create the transfer as shown in Figure 7 (a) and (b), respectively. This process was effective but possessed limitations including having to fabricate apertures to tune the shape as well as the necessity of changing apertures when a new design needed to be transferred onto the same substrate. The solution was to use a Digital Mirror Device, which is an array of micron scale mirrors that can be individually actuated. By utilizing this device, the imaged laser can be reconfigured on the fly, allowing for rapid production of systems with arrays of structures with different sizes and shapes while avoiding any changes to the optical setup's alignment [6]. An image of the beam profile after it has reflected of the DMD, and the resultant transfer can be seen in Figure 7 (c) and (d), respectively.

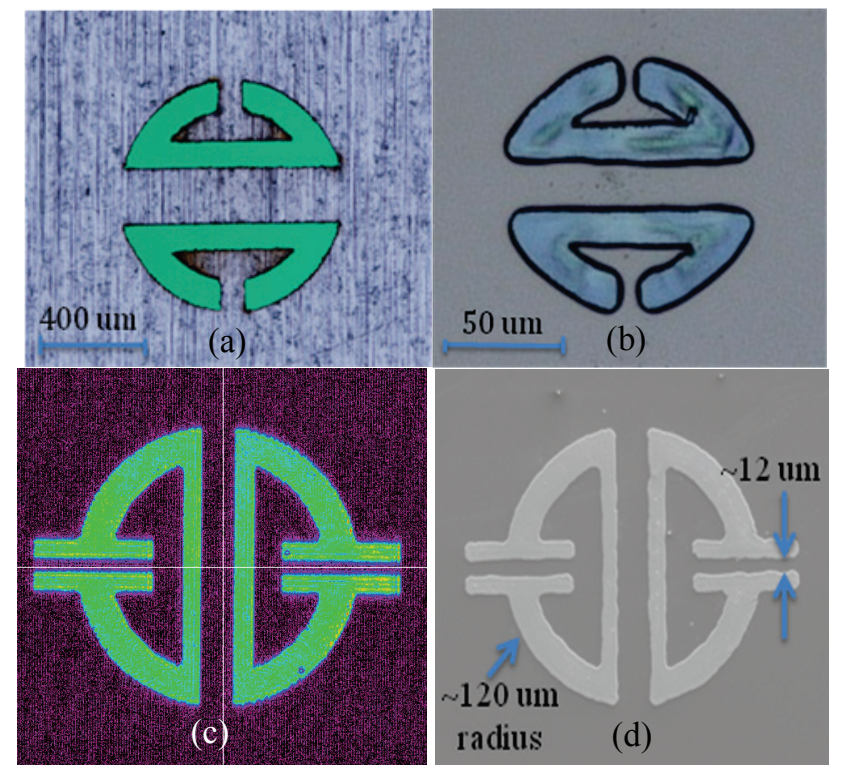

Figure 7 (a) An aperture fabricated to image the beam for LDT. (b) A D-Ring transferred using an aperture. (c) A beam profile image of the beam shaped by a DMD. (d) An SEM of a LDT DRing using a DMD.

\section{Results}

The fabrication of the $10 \mathrm{GHz}$ and $30 \mathrm{GHz}$ versions of the D-Ring and Crescent Rings, as well as the $2^{\text {nd }}$ iteration of the D-Ring, using LIFT showed good edge definition and feature resolution, qualitatively. After four passes, the thickness of the metal layer was approximately $2 \mu \mathrm{m}$. The key fabrication considerations and dimensions were all met within acceptable tolerances, particularly the interaction gap which was $10 \pm 1 \mu \mathrm{m}$ and the alignment which was within one micron from ideal alignment of the waveguide.

Figure 8 gives a high magnification view of the finished antenna, showing the very precise alignment with the underlying waveguide.

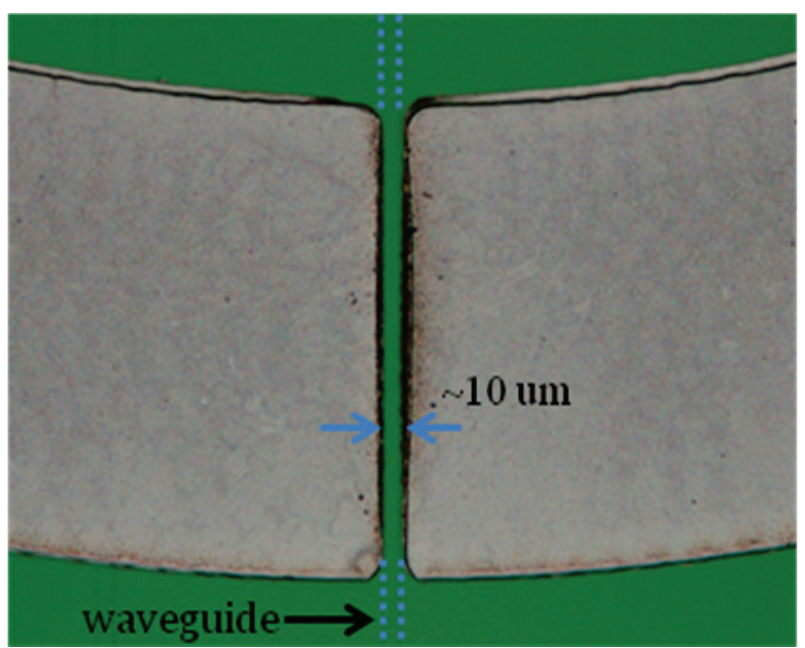

Figure 8 A finished D-Ring showing good edge and corner quality as well as alignment over the waveguide that is within $1 \mu \mathrm{m}$.

The LDT transferred D-Rings showed reasonable edge and corner definition. The design transferred using an aperture had an interaction gap of only $5 \mu \mathrm{m}$ while the DMD fabricated design had a gap of $12 \mu \mathrm{m}$. There were signs of rough edges for the DMD transfers and further studies are being done to determine whether this is a result of a resolution limit of the DMD.

Profilometry measurements of the overall thickness of devices made using both methods of fabrication are shown in Figure 9. The figure shows the different profiles that are a result of the different viscosities of each material. For the LIFT fabricated D-Rings, the top surface is curved, reflecting the wetting characteristics of the low viscosity ink. The LDT transfer, however, shows a much more flat surface with the exception of the sides which are stretched as a result of shearing deformation that is an effect of the high viscosity of the paste. For the LDT fabrication of the proposed higher frequency designs, the requirement for the thickness of the metal layer was relaxed since the skin depth for $\mathrm{W}$-band devices is much lower $(<300 \mathrm{~nm})$ than for X-band devices. The thickness of the LDT fabricated D-Rings was $400-500 \mathrm{~nm}$ after curing for a single pass. It is possible, however, to transfer thicker layers or to fire multiple shots and stack the structures on top of each other in order to build the height of the unit antenna.

Carrying out resistivity measurements on structures made using the same multi-step process yielded resistivity 
of approximately $5 \mathrm{u} \Omega / \mathrm{cm}$, which is nearly 3 times the resistivity of bulk silver.
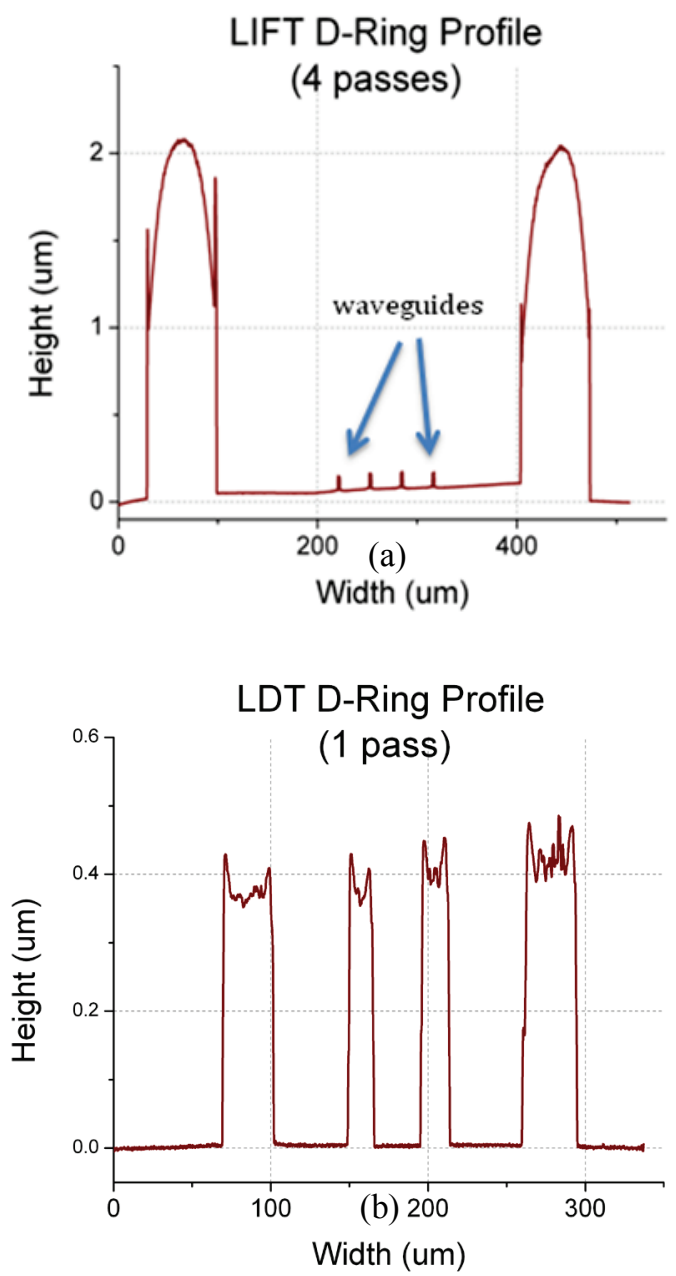

Figure 9 Thickness profiles of (a) a D-Ring fabricated using LIFT and (b) a D-Ring fabricated using LDT

The first iteration antenna designs fabricated using LIFT were tested and their field enhancement values were extrapolated using the known value of lithium niobates' electro-optic coefficient and the measured modulated optical signal strength. This field enhancement value was determined to be approximately 500 for the D-Ring antenna which was similar to antennas made using lithography. The Crescent Ring had enhancement value of approximately 700 , but its shorter interaction length made the modulated signal strength lower than for the D-Ring antennas.

The RF response of the second iteration of D-Ring antennas fabricated using LIFT was tested by placing a lithium niobate wafer with the fabricated antennas in a waveguide. The $\mathrm{S}_{11}$ measurements were then taken and the results are displayed in Figure 10. The resonance was not exactly at the desired $10 \mathrm{GHz}$ frequency, being closer to $9.4 \mathrm{GHz}$; however, all the peaks were nearly perfectly aligned, demonstrating excellent fabrication consistency and reproducibility. Furthermore, the performance of the Direct Write was similar to that of devices made from traditional lithography. RF measurements for the high frequency D-Rings are pending.

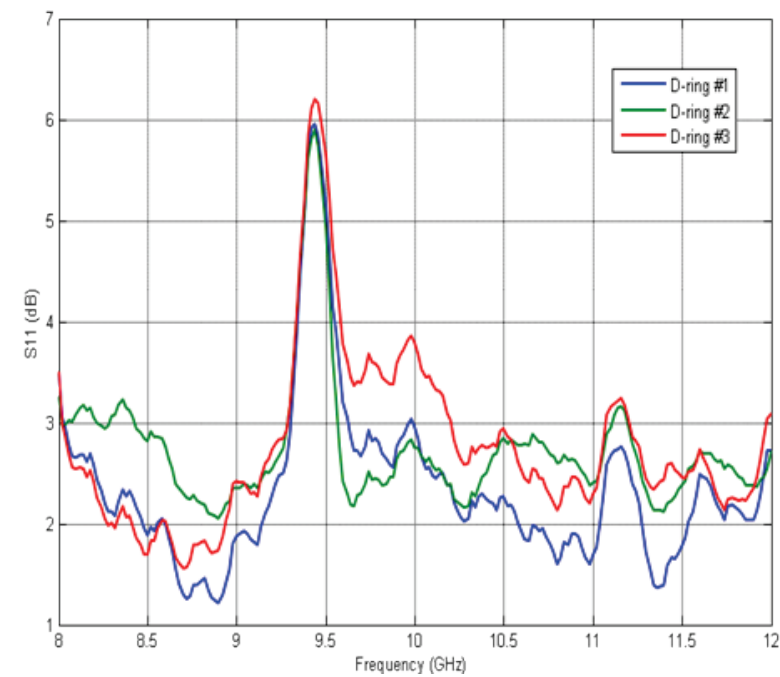

Figure $10 \mathrm{~S}_{11}$ measurements of the multiple $2^{\text {nd }}$ iteration antennas fabricated using LIFT

\section{Summary}

Laser Direct Write techniques were demonstrated to be viable for the fabrication of antennas that are capable of electro-optic conversion. Using a new process that allowed for thicker metal layers while simultaneously protecting the substrate surface, LIFT was utilized effectively in making working devices with performance comparable to those of antennas fabricated using traditional lithography. For higher frequency designs, LDT was implemented successfully to create the D-Ring structures that still fulfilled the design constraints. In addition to this, a Digital Mirror Device was used to image the beam for LDT, allowing for more rapid and flexible fabrication.

\section{Acknowledgments and Appendixes}

This work was supported by the Office of Naval Research and the Air Force Office of Scientific Research. Thanks to Dr. Shouyuan Shi at the University of Delaware for providing RF measurements of the antennas.

\section{References}

[1] W. Chen, R. L. Nelson and Q. Zhan: Proc. SPIE 7936, (2011) 79360E.

[2] H. Kim, R C Y Auyeung, S. H. Lee, A. L. Huston, and A. Piqué: Appl. Phys. A 96, (2009) 2314

[3] H. Kim, R C Y Auyeung, and A. Piqué: J. of Power Sources, 165, (2007) 413

[4] H. Kim, R C Y Auyeung, M. Ollinger, G. P. Kushto, Z H Kafafi, and A. Pique: Appl. Phys. A, 83, (2006) 73

[5] C.B. Arnold, P. Serra, and A. Piqué: MRS Bulletin, 32, (2007) 23-31.

[6] R. C. Y. Auyeung, H. Kim, N.A. Charipar A.J. Birnbaum, S.A. Mathews, A. Piqué: Appl Phys A 102, (2011), 21-26

[7] H. Stuart and R, Tran: Appl. Phys. Lett. 87, (2005) 151108 .

(Received: June 30, 2012, Accepted: September 25, 2012) 